\title{
The molecular condensations ahead of Herbig-Haro objects
}

\section{A theoretical investigation of the $\mathrm{HH} 2$ condensation}

\author{
S. Viti ${ }^{1,2}$, J. M. Girart ${ }^{3}$, R. Garrod ${ }^{1}$, D. A. Williams ${ }^{1}$, and R. Estalella ${ }^{3}$ \\ 1 Department of Physics and Astronomy, UCL, Gower St., London, WC1E 6BT, UK \\ 2 CNR-Istituto di Fisica dello Spazio Interplanetario, Area di Ricerca di Tor Vergata, via del Fosso del Cavaliere 100, \\ 00133 Roma, Italy \\ ${ }^{3}$ Department d'Astronomia i Meteorologia, Universitat de Barcelona, Av. Diagonal 647, 08028 Barcelona, Catalunya, Spain
}

\author{
Received 22 October 2002 / Accepted 22 November 2002
}

\begin{abstract}
Clumps of enhanced molecular emission are present close to a number of Herbig-Haro (HH) objects. These enhancements may be the consequence of an active photochemistry driven by the UV radiation originating from the shock front of the $\mathrm{HH}$ object. On the basis of this picture and as a follow up to a molecular line survey toward the quiescent molecular clump ahead of the HH object, HH 2 (Girart et al. 2002), we present a detailed time and depth dependent chemical model of the observed clump. Despite several difficulties in matching the observations, we constrain some of the physical and chemical parameters of the clump ahead of HH 2. In particular, we find that the clump is best described by more than one density component with a peak density of $3 \times 10^{5} \mathrm{~cm}^{-3}$ and a visual extinction of $\leq 3.5 \mathrm{mag}$; its lifetime can not be much higher than 100 years and the impinging radiation is enhanced with respect to the ambient one by probably no more than 3 orders of magnitude. Our models also indicate that carbon-bearing species should not completely hydrogenate as methane when freezing out on grains during the formation of the clump.
\end{abstract}

Key words. ISM: clouds - ISM: molecules - ISM: general

\section{Introduction}

Herbig-Haro (HH) objects are regions of hot plasma created by stellar jets impacting on interstellar gas. In the last decade, molecular condensations have been found ahead of $\mathrm{HH}$ objects. These clumps were detected in enhanced emission from $\mathrm{HCO}^{+}$ and $\mathrm{NH}_{3}$ (e.g. Rudolph \& Welch 1988; Torrelles et al. 1992; Girart et al. 1994). The detected clumps are cool, with temperatures around $10 \mathrm{~K}$, and quiescent: they show no evidence of shocks and are therefore not connected dynamically with the stellar jet. The enhanced emission comes from localised regions in the vicinity of the $\mathrm{HH}$ objects, and has now been detected from a number of objects (Girart et al. 2002 (hereafter Paper I), and references therein). Torrelles et al. (1992) suggested that the chemical enhancements may be explained by UV radiation from the HH object; Girart et al. (1994) added that the mechanism induced by the UV radiation could be the removal of the ice mantles from grains in the clumps ahead of the jet which then would drive a photochemistry. In this picture, the jet and its $\mathrm{HH}$ object constitute a micro-probe of the physical and chemical nature of the molecular cloud. Theoretical studies (Taylor \& Williams 1996; Viti \& Williams 1999, hereafter VW99) supported this scenario and also showed that

Send offprint requests to: S. Viti, e-mail: sv@star.ucl.ac.uk the chemistry of these clumps should be rich; the impact of a high radiation field on a dense gas in a clump should yield enhanced abundances not only of $\mathrm{HCO}^{+}$and $\mathrm{NH}_{3}$ but also of several other species such as $\mathrm{CH}_{3} \mathrm{OH}, \mathrm{H}_{2} \mathrm{~S}, \mathrm{H}_{2} \mathrm{CO}$, SO and $\mathrm{SO}_{2}$. Also, as the jet is moving at quite high speeds (100$1000 \mathrm{~km} \mathrm{~s}^{-1}$ ), the clumps ahead of these jets must be transient in that they will eventually be reached by the jet and may be destroyed within a timescale on the order of 1000 years or less (Raga \& Williams 2000). As a consequence, considering their short lifetime, statistically, these clumps must be very common.

Understanding the nature of the condensations ahead of $\mathrm{HH}$ objects can therefore shed light on the nature of the interstellar molecular clouds. The condensations associated with HH objects appear to have the same physical characteristics as transient dense clumps believed to be the birthplace of lowmass stars. The physical and chemical nature of these clumps are usually explored via the detection of emission in several molecules that trace dense gas such as $\mathrm{NH}_{3}$ and CS. However, low angular resolution contour maps of molecular emission of these two species are puzzling: the CS half-power contour is much more extended than that of ammonia, their peaks are displaced, and the widths of their lines are different (Pastor et al. 1991; Myers et al. 1991; Morata et al. 1997). The $\mathrm{NH}_{3}$ contour is closely associated with embedded sources in the cloud. 
One interpretation of these differing morphologies is that the cloud is composed of transient clumps, unresolved in these observations, that develop CS as the chemistry evolves, but disperse before appreciable $\mathrm{NH}_{3}$ is formed (Taylor et al. 1996). Occasionally, a more massive clump will evolve faster - both chemically and dynamically - and so would be responsible for the spatial association of $\mathrm{NH}_{3}$ with star formation signatures. Observational support for this model is now provided by high resolution BIMA observations in lines of CS, $\mathrm{HCO}^{+}$, and $\mathrm{N}_{2} \mathrm{H}^{+}$ of a portion of the molecular cloud L673 (Morata et al. 2003). These observations show much structure on scales of 10 arcsec, different in the different species, suggesting that the structure is transient and has time-dependent chemistry.

We suggest that the clumps ahead of the HH objects may be of the same population as the clumps observed by Morata et al. (2003) but they happen to have a different chemistry because they are illuminated by - and are photoprocessed by - the $\mathrm{HH}$ radiation. Hence, observations of the condensations associated with $\mathrm{HH}$ objects give an independent method of determining physical parameters of dense clumps in interstellar molecular clouds.

Following theoretical studies which indicated that the impact of radiation from an $\mathrm{HH}$ object on a dense gas should lead to a rich chemistry (Taylor et al. 1996; VW99) a first detailed line survey of a condensation ahead of an $\mathrm{HH}$ object $(\mathrm{HH} 2$ : Herbig 1951; Haro 1952) was made (Paper I): the condensation ahead of $\mathrm{HH} 2$ was first established by detections of enhanced $\mathrm{HCO}^{+}$and $\mathrm{NH}_{3}$ (Davis et al. 1990; Torrelles et al. 1992, 1994; Choi \& Zhou 1997). In Paper I we presented CSO and BIMA detections of 14 species and we derived the chemical composition of the clump. We also briefly compared the observational results with the VW99 models and found a qualitative agreement. This comparison helped to constrain some of the parameters of this clump such as its maximum age (for which we found $\sim 1000 \mathrm{yrs}$ ). However, the VW99 models were not specific to $\mathrm{HH} 2$ : the abundances at any one time depended on several initial parameters such as the strength of the radiation field, the fraction of gas frozen on to the grains as ices, the age and size of the clump, and the initial composition of the gas. As a consequence, we were not able to constrain important physical parameters such as the radiation field strength and whether the observed chemistry was a direct consequence of the vicinity of the HH 2 knot. Moreover, it is now established that the strongest HH 2 knot, which is also the highest excitation knot, has associated X-ray emission (Pravdo et al. 2001). This may affect the chemistry and needs to be taken into consideration. A more detailed model specific to $\mathrm{HH} 2$ is therefore needed.

The aim of this work is to develop a detailed model of the chemistry in the clump associated with $\mathrm{HH} 2$ and to use it to determine the chemical and physical parameters of the $\mathrm{HH} 2$ clump. In Sect. 2 we summarize the observational situation, and in Sect. 3 we describe the models used in the calculations. Section 4 gives results of calculations for many parameter variations; these results are discussed in Sect. 5, and our conclusions are listed.

\section{Physical and chemical parameters of the clump ahead of HH 2 - the present status of observational studies}

In Paper I we derived column densities from a 0.8-3 mm molecular line survey toward the quiescent molecular clump ahead of the bright HH 2 object. For simplicity, we list again here the column densities observed (Table 1). Note that from more recent observations with the IRAM $30 \mathrm{~m}$ telescope (in preparation) we derive a column density for $\mathrm{HCO}^{+}$which is one order of magnitude less than the one derived in Paper I. This difference is probably due to the different beam sizes (CSO $\sim 30^{\prime \prime}$; IRAM $\sim 21^{\prime \prime}$ ) and different pointing positions, in a region where density and chemistry both vary strongly with position. This point will be further discussed in a future paper. Here we only draw the attention on the possible range of values for the abundance of $\mathrm{HCO}^{+}$.

In particular, from the morphology of the emission we find three groups of species (Girart et al. 2003, Paper III, in prep.):

- SO, $\mathrm{SO}_{2}$ and $\mathrm{CH}_{3} \mathrm{OH}$ with compact emission just ahead of the $\mathrm{HH}$ object;

- $\mathrm{HCO}^{+}, \mathrm{HCN}$ and $\mathrm{H}_{2} \mathrm{CO}$ with a strong compact clump just ahead of the $\mathrm{HH}$ object surrounded by more diffuse and extended emission north-east of the compact clump;

- $\mathrm{CS}$ and $\mathrm{C}^{18} \mathrm{O}$ with a peak intensity position clearly offset from that of the other molecules.

Chemically, this may mean that $\mathrm{SO}, \mathrm{SO}_{2}$ and $\mathrm{CH}_{3} \mathrm{OH}$ are heavily affected by the HH 2 knot (by the UV flux or the X-ray emission or a combination of both) and they are probably very low in abundance in the interclump medium; $\mathrm{HCO}^{+}, \mathrm{HCN}$ and $\mathrm{H}_{2} \mathrm{CO}$ are also affected by the $\mathrm{HH} 2$ object and this effect shows up as a molecular intensity enhancement with respect to the interclump medium, where these species are however already present in detectable quantities. Finally $\mathrm{CS}$ and $\mathrm{C}^{18} \mathrm{O}$ seem to be largely unaffected by the vicinity of the HH 2 knot, or maybe even depleted (e.g. by photodissociation) just ahead of the $\mathrm{HH}$ object.

There is a large gradient of abundances for most of the species which indicate a complex structure. This is clear from Fig. 1 where the column densities deduced from BIMA observations of selected species as a function of distance to the $\mathrm{HH} 2$ knot are shown. By taking into account the column densities listed in Table 1, and the gradient of values from Fig. 1, we now investigate whether we are able to reproduce both the observed column densities and the spatial distribution and chemical differentiation shown in the BIMA maps.

\section{The calculations}

\subsection{The model}

The model we adopt is an extension of the model employed in VW99. The model calculation is carried out in two phases; both are time- and depth-dependent. In Phase I, the clump is represented by a region that collapses gravitationally within the molecular cloud. The collapse is treated as a free-fall, as described by Rawlings et al. (1992). The effects of a retarded 
Table 1. Extract of Table 3 from Paper I: column densities of observed species.

\begin{tabular}{cc}
\hline \hline Molecule & Column density \\
\hline $\mathrm{CO}$ & $3.8(17)$ \\
$\mathrm{HCO}^{+}$ & $1.0(13)-1.0(14)^{*}$ \\
$\mathrm{CH}_{3} \mathrm{OH}$ & $6.4(13)$ \\
$\mathrm{H}_{2} \mathrm{CO}$ & $5.7(13)$ \\
$\mathrm{SO}$ & $3.2(13)$ \\
$\mathrm{SO}_{2}$ & $1.7(13)$ \\
$\mathrm{HCN}$ & $4.1(12)$ \\
$\mathrm{CN}$ & $3.4(12)$ \\
$\mathrm{CS}$ & $2.8(12)$ \\
\hline
\end{tabular}

${ }^{*}$ The low value is a lower limit derived from IRAM observations. See text.

collapse have already been discussed (VW99). During the collapse phase, gas-phase chemistry and freeze-out on to dust grains with subsequent processing are assumed to occur. The initial density of the clump is taken to be $1000 \mathrm{~cm}^{-3}$, and the final density is treated as a free parameter. In Phase II we simulate the presence of the $\mathrm{HH}$ object by subjecting the clump to the $\mathrm{HH}$ radiation field (which evaporates all the ices and promotes a photochemistry in what was formerly dark cloud material) and also to the X-ray flux. The chemical network is taken from the UMIST database (Millar et al. 1997) and is described in VW99. We refer the reader to VW99 for a detailed explanation of the model, to which the following changes have been made for the present study:

i) In Phase II, we simulate crudely the presence of X-ray ionization by enhancing our standard cosmic ray ionization rate, $\zeta\left(1 \zeta=1.3 \times 10^{-17} \mathrm{~s}^{-1}\right)$, as in Nguyen et al. (2002).

ii) In Phase II, the enhanced radiation field is now depthdependent $\left(\propto 1 / r^{2}\right.$, where $r$ is the distance from the $\mathrm{HH}$ shock). The constant of proportionality is decided by imposing a value (variable) for the radiation field at the edge of the clump.

\subsection{The model parameter grids}

We have computed two separate grids of models (Grids A and $\mathrm{B}$ ), differing in the assumption of the age of the clump at the end of Phase I. The results from these two grids will be discussed separately in Sects. 4.1 and 4.2. For Grid A Models, we assumed that once the final density was reached (between $3 \times 10^{5} \mathrm{yrs}$ and $\left.4.2 \times 10^{5} \mathrm{yrs}\right)$, the clump survived for a substantial amount of time ( $\sim 2$ Myrs) before being affected by the $\mathrm{HH}$ object. This is different from our assumption in VW99, and from Grid B (see below), where we assumed that once the final density was reached the clump was immediately irradiated.

In order to determine the parameter space needed to be investigated, we recall the observational results from Papers I and III: the temperature of the clump is estimated to be $\sim 13 \mathrm{~K}$ and the upper limit to the hydrogen molecule number density is $\sim 3 \times 10^{5} \mathrm{~cm}^{-3}$. The size of the clump is derived from the SO contour, giving a clump diameter of $\sim 0.01 \mathrm{pc}$. This corresponds to a visual extinction of about 6 mag with respect

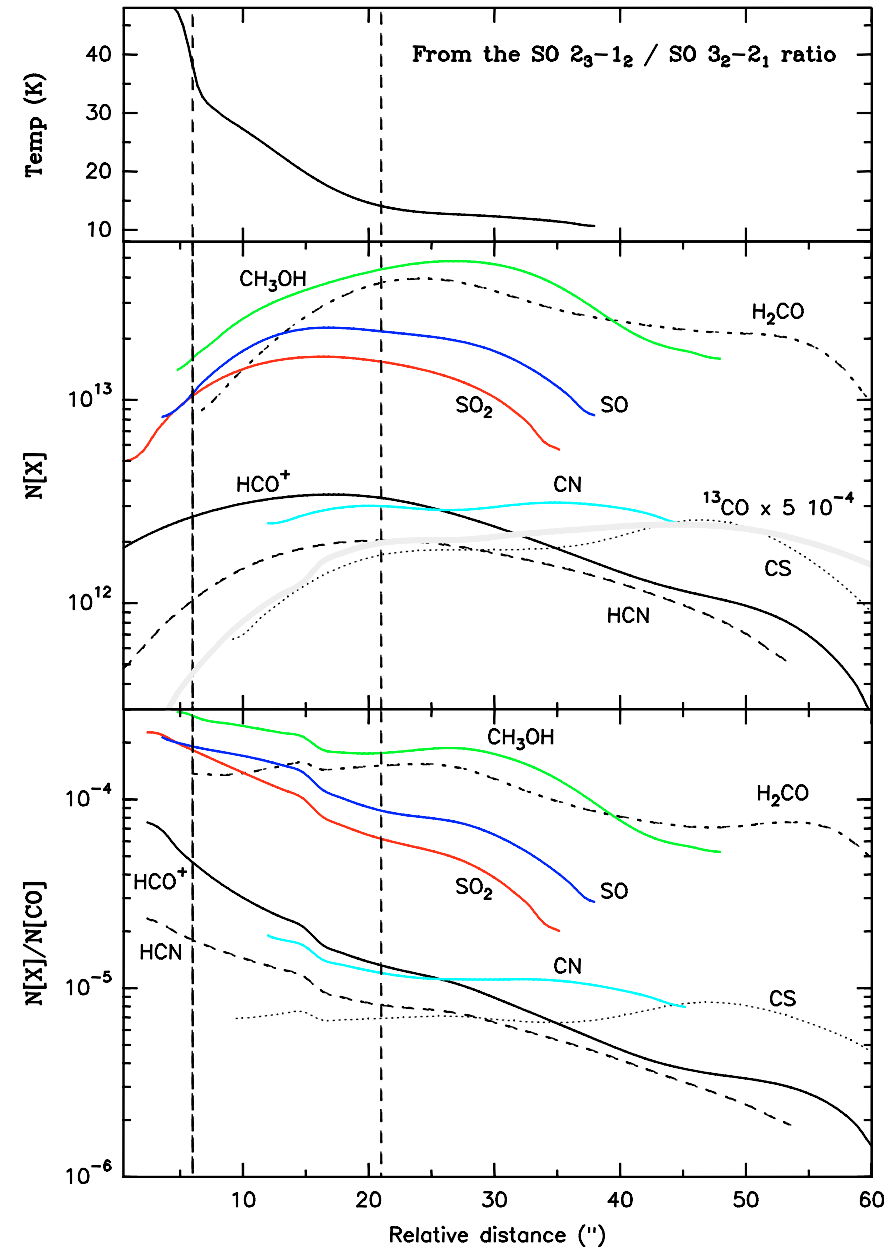

Fig. 1. Slices of the rotational temperature (top panel), column density (central panel) and relative abundance with respect to the $\mathrm{CO}$ (bottom panel) as a function of distance to $\mathrm{HH} 2$. The left vertical dashed line shows the position of HH 2 knot L, while the right dashed line shows approximately the position where the CSO spectrum was taken (Paper I). The slice has a 0 position $\mathrm{RA}(\mathrm{J} 2000)=$ 05:36:26.55, $\operatorname{Dec}(\mathrm{J} 2000)=-06: 47: 06.0$ and the position angle of the slice is $\mathrm{PA}=169$.

to the position of the $\mathrm{HH}$ object. However, this inferred extinction across the clump assumes that the SO contour corresponds to the true size of the clump and the gas around the clump is completely deficient in SO; and that the material between us and the clump along the line of sight is diffuse. All these assumptions are uncertain, and we therefore treat the clump as a one-dimensional multi-point slab extending up to 6.4 mag and examine the time-dependent chemistry throughout the clump. We consider lower and upper limits to the density of $3 \times 10^{4}$ and $3 \times 10^{5} \mathrm{~cm}^{-3}$, respectively.

We assume that, during Phase I, a fraction of the gas phase material is frozen on to the grains, and undergoes hydrogenation, where this is chemically possible. This fraction is arranged by adjusting the grain surface area per unit volume, and assumes a sticking probability of unity for all species. The fraction of material on grains is then dependent on the product of the sticking probability and the amount of cross section provided per unit volume by the adopted grain size distribution. 


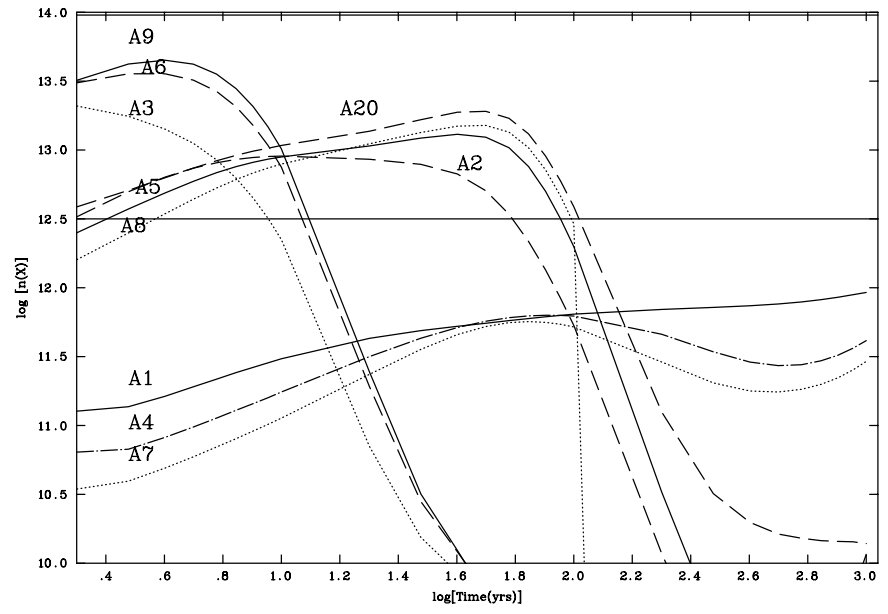

Fig. 2. Column density of $\mathrm{HCO}^{+}$as a function of time at $A_{v}=3.4 \mathrm{mag}$ for selected models from Grid A. The two horizontal lines encompass the area of possible observed values for $\mathrm{HCO}^{+}$.

This product was varied so that at the end of Phase I, we would have a low, medium or high abundance of ices (see Col. 3 of Table 2). Note that as the chemistry is time-dependent, different species form at different times. As a consequence the material frozen out on the grains at any time is not representative of the whole gas but of selected species. In this case, we have determined the fraction of material frozen out by monitoring mainly the amount of frozen $\mathrm{CO}$, and $\mathrm{H}_{2} \mathrm{O}$ at the edge and centre of the clump.

During Phase II, we investigated the chemical consequences of changes in the following parameters: firstly, the strength of the X-ray emission by enhancing the cosmic ray ionisation rate up to 1000 times its canonical value; and, secondly, the strength of the radiation field impinging on the edge of the clump ( $\chi_{\text {edge }}$ ) by varying it from 20 to $10^{4}$ times the mean interstellar radiation intensity, here called Habing units (Habing 1968). This latter parameter is of paramount importance as it is completely unconstrained by direct observations of the $\mathrm{HH} 2$ object from which only the $\mathrm{H}_{2}$ line flux can be derived. Table 2 gives the parameters defining most of the models computed.

\section{Results}

\subsection{Long-lived clumps: Models A}

In order to investigate whether it is feasible for these condensations to be long-lived before they are illuminated by the $\mathrm{HH}$ radiation we compute a grid of models (Grid $\mathrm{A}$ ) where the chemistry is allowed to continue after the clump has reached the desirable final density. The column densities estimated from Grid A Models are shown in Table 3 and the $\mathrm{HCO}^{+}$abundance as a function of time for selected Grid A models is shown in Fig. 2.

Briefly, we discuss the trends evident in the table and figure. Note that from the BIMA maps we believe that the geometry of the clump we observed is such that our line of sight is almost perpendicular to the direction of the radiation coming from the $\mathrm{HH}$ object (Paper III) - this means that our
Table 2. Model parameters. The notation $a(b)$ signifies $a \times 10^{b}$. The model number is listed in Col. 1; Col. 2 shows the density of the gas; Cols. 3, 4 and 5 show respectively the percentage of the gas depleted onto grain at the end of Phase I, the enhancement factor of the radiation field in units of Habing and enhancement factor of the ionization rate, in units of $\zeta$.

\begin{tabular}{|c|c|c|c|c|}
\hline Model & $n_{f}\left(\mathrm{~cm}^{-3}\right)$ & FR (\%) & $\chi$ (Habing) & $\zeta\left(\mathrm{s}^{-1}\right)$ \\
\hline \multicolumn{5}{|c|}{ Grid A } \\
\hline A1 & $3(5)$ & 15 & 20 & 1 \\
\hline $\mathrm{A} 2$ & $3(5)$ & 15 & $1(3)$ & 1 \\
\hline A3 & $3(5)$ & 15 & $1(4)$ & 1 \\
\hline A4 & $3(5)$ & 60 & 20 & 1 \\
\hline A5 & $3(5)$ & 60 & $1(3)$ & 1 \\
\hline A6 & $3(5)$ & 60 & $1(4)$ & 1 \\
\hline A7 & $3(5)$ & 80 & 20 & 1 \\
\hline A 8 & $3(5)$ & 80 & $1(3)$ & 1 \\
\hline A9 & $3(5)$ & 80 & $1(4)$ & 1 \\
\hline A10 & $3(5)$ & 60 & 20 & 100 \\
\hline A11 & $3(5)$ & 60 & 20 & $1(3)$ \\
\hline A12 & $3(4)$ & 20 & 20 & 1 \\
\hline A13 & $3(4)$ & 20 & $1(3)$ & 1 \\
\hline A14 & $3(4)$ & 20 & $1(4)$ & 1 \\
\hline A15 & $3(4)$ & 40 & 20 & 1 \\
\hline A16 & $3(4)$ & 40 & $1(3)$ & 1 \\
\hline A17 & $3(4)$ & 40 & $1(4)$ & 1 \\
\hline A18 & $3(4)$ & 40 & 20 & 100 \\
\hline A19 & $3(4)$ & 40 & 20 & $1(3)$ \\
\hline A20 & $3(5)$ & 60 & $1(3)$ & 100 \\
\hline \multicolumn{5}{|c|}{ Grid B } \\
\hline B1 & $3(5)$ & $7 \%$ & 20 & 1 \\
\hline B2 & $3(5)$ & $20 \%$ & 20 & 1 \\
\hline B3 & $3(5)$ & $55 \%$ & 20 & 1 \\
\hline B4 & $3(5)$ & $0 \%$ & 20 & 1 \\
\hline B5 & $3(5)$ & $0 \%$ & 1 & 1 \\
\hline B6 & $3(5)$ & $7 \%$ & $1(3)$ & 1 \\
\hline B7 & $3(5)$ & $20 \%$ & $1(3)$ & 1 \\
\hline B8 & $3(5)$ & $55 \%$ & $1(3)$ & 1 \\
\hline B9 & $3(5)$ & $20 \%$ & $1(4)$ & 1 \\
\hline B10 & $3(5)$ & $20 \%$ & 1 & 1 \\
\hline
\end{tabular}

column densities need to be estimated as we did in VW99 (i.e. $10^{15}\left(X_{i} / 10^{-6}\right) \mathrm{cm}^{-2}$ for $\left.A_{v} \sim 1\right)$ rather than calculated by integrating along the depth points (which represent a slab perpendicular to our line of sight, along the radiation field line). From Fig. 2, we note already that most Grid A models fail to reproduce the high abundance of $\mathrm{HCO}^{+}$for long enough time, although models A2, A5, A8 and A20 do succeed in matching the $\mathrm{HCO}^{+}$lower limit. From Table 3, we note that most of the species are affected by an enhanced radiation field. At early times $\mathrm{HCO}^{+}$increases when we increase the impinging radiation field. However, we note that at late times (not shown), it is lower when the radiation field is higher. This effect is more pronounced at low-to-intermediate visual extinctions ( $\left.A_{v} \leq 4 \mathrm{mag}\right)$. CS, on the other hand, seems to decrease slightly with an increase in radiation when the freeze-out is low but increases when the latter is high. SO behaves in a similar way to CS but at low freeze out, when we increase the 
Table 3. Column densities for selected species for Models A1-A9 and A20 at 3 years after the clump has been irradiated, at $A_{V}=3.4$ mag.

\begin{tabular}{ccccccccccc}
\hline \hline & $\mathrm{A} 1$ & $\mathrm{~A} 2$ & $\mathrm{~A} 3$ & $\mathrm{~A} 4$ & $\mathrm{~A} 5$ & $\mathrm{~A} 6$ & $\mathrm{~A} 7$ & $\mathrm{~A} 8$ & A9 & A20 \\
\hline $\mathrm{CO}$ & $9.1(17)$ & $9.2(17)$ & $9.4(17)$ & $6.0(17)$ & $6.1(17)$ & $6.4(17)$ & $4.5(17)$ & $4.5(17)$ & $5.0(17)$ & $6.1(17)$ \\
$\mathrm{HCO}^{+}$ & $1.4(11)$ & $5.0(12)$ & $1.8(13)$ & $6.7(10)$ & $3.7(12)$ & $3.6(13)$ & $3.9(10)$ & $2.5(12)$ & $4.2(13)$ & $5.1(12)$ \\
$\mathrm{H}_{2} \mathrm{CO}$ & $9.4(13)$ & $1.1(15)$ & $2.5(15)$ & $1.2(14)$ & $1.4(15)$ & $4.8(15)$ & $1.5(14)$ & $1.3(15)$ & $5.8(15)$ & $1.4(15)$ \\
$\mathrm{CS}$ & $3.5(13)$ & $3.4(13)$ & $2.3(13)$ & $6.3(12)$ & $7.7(12)$ & $1.3(13)$ & $1.5(12)$ & $2.4(12)$ & $1.1(13)$ & $7.7(12)$ \\
$\mathrm{SO}$ & $3.1(12)$ & $2.9(13)$ & $2.2(13)$ & $5.8(12)$ & $2.4(13)$ & $3.7(13)$ & $4.6(12)$ & $1.8(13)$ & $4.2(13)$ & $2.4(13)$ \\
$\mathrm{SO}_{2}$ & $3.7(12)$ & $3.3(12)$ & $1.4(12)$ & $4.5(12)$ & $4.2(12)$ & $3.5(12)$ & $3.4(12)$ & $3.3(12)$ & $4.4(12)$ & $4.2(12)$ \\
$\mathrm{CN}$ & $1.4(12)$ & $4.7(13)$ & $5.6(14)$ & $1.0(12)$ & $4.2(13)$ & $1.2(15)$ & $9.8(11)$ & $4.0(13)$ & $1.5(15)$ & $4.1(13)$ \\
$\mathrm{HCN}$ & $7.3(13)$ & $9.5(13)$ & $1.6(14)$ & $6.1(13)$ & $8.4(13)$ & $2.4(14)$ & $5.8(13)$ & $8.0(13)$ & $2.8(14)$ & $8.2(13)$ \\
$\mathrm{CH}_{3} \mathrm{OH}$ & $3.3(15)$ & $2.7(15)$ & $5.6(14)$ & $4.1(15)$ & $3.4(15)$ & $1.3(15)$ & $3.6(15)$ & $3.0(15)$ & $1.6(15)$ & $3.4(15)$ \\
\hline
\end{tabular}

impinging radiation from 20 to 1000 it actually slightly increases. $\mathrm{SO}_{2}$ decreases in abundance with an increase in radiation at low and intermediate freeze out but increases when we increase $\chi$ from 20 to 1000 at high freeze out (but decreases again when $\chi$ is 10000). At early times, $\mathrm{CN}$ always seems to increase slightly with radiation while $\mathrm{CH}_{3} \mathrm{OH}$ decreases with increasing radiation, regardless of the visual extinction or age; $\mathrm{HCN}$ shows the same behaviour of early time $\mathrm{HCO}^{+}$, but at all ages. At first sight, it seems that the enhanced radiation field in the vicinity of a $\mathrm{HH}$ object cannot be directly responsible for a molecular enhancement which survives for a long enough time to be observed.

One of the most significant parameters affecting the results is the fraction of gas frozen-out and hydrogenated on the grains by the end of Phase I. We find that many of the species, including $\mathrm{HCO}^{+}$, always decrease in abundance with an increase of freeze out.

These two trends are apparently inconsistent with our previous models (VW99) and with the explanation that the radiation generated in the HH shock affects the clumps ahead of it by evaporating the icy mantles. However, a close examination of the $\mathrm{HCO}^{+}$formation network shows that the main characteristic in this set of Models $\mathrm{A}$ is the lack of $\mathrm{C}^{+}$: although $\mathrm{H}_{2} \mathrm{O}$ ice is abundant (due to the hydrogenation of oxygen bearing species when they freeze out), $\mathrm{C}^{+}$is very low. This is due to a much lower abundance of neutral carbon at the end of Phase I since this phase is of a long duration. When $\mathrm{C}$ freezes out, it hydrogenates into methane. This effect is also more pronounced for models where the density is high $\left(3 \times 10^{5} \mathrm{~cm}^{-3}\right)$. For Grid A Models, once the collapse is stopped and the final density is reached the clump continues to chemically evolve for a long time at high density, and the freeze-out and hydrogenation efficiency adopted assures that most of the carbon is locked in frozen methane.

This effect is partly reduced by simulating the effect of $\mathrm{X}$-rays. The primary effect of the increase in the ionisation rate is to raise the abundances of various ionised species (mostly single atoms) compared to their neutral abundances. This effect is most obvious at high $A_{v}$, where an enhanced ionisation rate is the only significant contributor to ionisation, since the radiation field is heavily suppressed. However, the increase in the ionisation rate also causes more dissociation of molecules. The increased dissociation of CO causes a significant increase in $\mathrm{C}$ at high $A_{v}$, where the abundance of $\mathrm{C}$ is lowest, and this in turn allows higher values of $\mathrm{C}^{+}$via ionization of $\mathrm{C}$. The $\mathrm{C}^{+}$may then readily react with $\mathrm{H}_{2} \mathrm{O}$, which at early times is abundant at all depths due to evaporation of icy mantles, enhancing $\mathrm{HCO}^{+}$. After 1000 years, ion-neutral reactions and photodissociation have greatly reduced the abundances of most molecules at lower $A_{v}$, for all values of the ionisation rate. In the case where the ionisation rate is 100 times the canonical value, at high $A_{V}$ the increased ionization causes considerable losses in the most abundant molecules. $\mathrm{CO}$, which is already very abundant, is the main beneficiary of the destruction of $\mathrm{H}_{2} \mathrm{O}$ and $\mathrm{CH}_{4}$. Increasing the ionisation rate to 1000 times the canonical value makes the increases in $\mathrm{CO}$ at large $A_{v}$ much greater, and abundances of $\mathrm{H}_{2} \mathrm{O}$ and $\mathrm{CH}_{4}$ fall correspondingly.

In general, $\mathrm{HCO}^{+}$is seen to be enhanced with the simulated X-ray field at large $A_{v}$, while other species such as $\mathrm{CH}_{3} \mathrm{OH}$ are not strongly affected, although at very high ionisation rates their abundances are reduced. Sulphur-bearing species are not affected at low $A_{V}$ but they increase, as a consequence of the faster destruction of $\mathrm{H}_{2} \mathrm{~S}$, at high $A_{v}$. In summary, simulating an X-ray field by enhancing the ionization rate has some effect on the chemistry of the clump. However, the consequences are not significant when compared to other effects.

From this first set of models we conclude that if the quiescent clump is indeed long-lived before the jet arrives, then the suggestion that these regions of enhanced emission arose because of the influence of $\mathrm{HH}$ radiation on the dense clump is not correct. A requirement for this theory to be correct is that we have a reasonable amount of free carbon in the gas phase when the jet gets close enough to irradiate the clump; we find that if the clump is long-lived, then either most of the carbon is locked in frozen $\mathrm{CH}_{4}$ or it is locked in $\mathrm{CO}$ (assuming a very low freeze out parameter). We therefore assume in what follows that - in general - high density clumps in interstellar clouds are transient. This is indeed consistent with the picture proposed by Taylor et al. (1996), with the observational evidence of Girart et al. (2002), and with the possible origin of such transient clumps in slow-mode MHD waves (Falle \& Hartquist (2002) - see Sect. 1.

Although Models A have not provided a match to the observations of HH 2 they have been instructive. Their results have demonstrated that the chemistry is extremely sensitive to variations in the density, to the fraction of gas frozen out as ices, and to lifetime of the clumps. We decided therefore to perform 
a second (reduced) grid of models (grid B) where Phase I is ended and Phase II is started as soon as the collapse is finished.

\subsection{Transient clumps: Models $B$}

Details of the grid of Models B are listed in Table 2. We note that for Grid B we have also evaluated a model in which species do not freeze out on to dust grains (Model B4), a model like Model B4 but where the radiation field in Phase II is kept equal to the ambient one (Model B5), and a model where freeze-out occurs but in which there is no enhancement in the ambient radiation field (Model B10). Other models were also computed, and we shall discuss them later.

$\mathrm{HCO}^{+}$is one of the molecules most enhanced in abundance in this clump; yet it is difficult to make in large amounts in the conditions found in clumps ahead of $\mathrm{HH}$ objects. Therefore, we first concentrate on this species. In Fig. 3 we show the column density of $\mathrm{HCO}^{+}$as a function of time for $A_{v} \sim 3$ (left plot) and $\sim 6$ mag (right plot). From Table 1 and Fig. 1 we derive a column density for $\mathrm{HCO}^{+}$between $3 \times 10^{12} \mathrm{~cm}^{-2}$ (indicated by the $\mathrm{HCO}^{+} 1-0$ emission not corrected by optical depth from BIMA (see Fig. 1) and IRAM observations) and $10^{14} \mathrm{~cm}^{-2}$ (obtained from the multi-transition analysis from BIMA and CSO observations) depending on where around the $\mathrm{HH}$ object we look. These values are shown in Fig. 3 (left and right panels) as the area encompassed between the two horizontal lines. From both plots we can see that it is quite difficult to reproduce a high abundance of $\mathrm{HCO}^{+}$. It certainly seems that, at high $A_{V}$, $\mathrm{HCO}^{+}$is never abundant. We can also definitely exclude models where freeze-out is not included but there is enhanced radiation (B4), models in which freeze-out is not included and there is no enhancement of the radiation field (B5), and models in which freeze-out $i s$ included but there is no enhancement of the radiation field (B10). These conclusions are consistent with the assumption that the enhanced chemistry of the condensation ahead of the HH 2 object is a direct consequence of evaporation of ices together with a photochemistry caused by the enhanced radiation field due to the proximity of the $\mathrm{HH}$ object. These conclusions confirm that there seems to be no alternative way of producing a high abundance of $\mathrm{HCO}^{+}$.

Another result from Fig. 3 is that high abundances of $\mathrm{HCO}^{+}$ are not easily maintained beyond about one hundred years. This period is, in fact, comparable to the expected survival time of the clump ahead of HH 2 (see Sect. 1). From these plots, the best model candidates are Models B6, B7 and B8 (see Table 2), for $A_{V} \leq 3.5 \mathrm{mag}$. They are favoured over Models A2, A5, and A8 in that they succeed in maintaining a higher $\mathrm{HCO}^{+}$abundance for a longer period.

Having found the models that match reasonably well the $\mathrm{HCO}^{+}$observations, we now investigate whether other species are also reasonably accounted for by these same models. While most of the species are well represented by B7 and B8, we find that some of the species, in particular $\mathrm{H}_{2} \mathrm{CO}, \mathrm{CN}$, and $\mathrm{HCN}$ are always overabundant (up to 2 orders of magnitude) in all our models. We now consider the chemistry of these species in an attempt to resolve these problems.
The chemistry of $\mathrm{H}_{2} \mathrm{CO}$, and to a lesser extent $\mathrm{CN}$ and $\mathrm{HCN}$, is indirectly governed by the abundance of $\mathrm{CH}_{4}$. The main reaction forming $\mathrm{H}_{2} \mathrm{CO}$ is:

$\mathrm{CH}_{3}+\mathrm{O} \rightarrow \mathrm{H}_{2} \mathrm{CO}+\mathrm{H}$

where $\mathrm{CH}_{3}$ comes from the photodissociation of $\mathrm{CH}_{4}$. We note that $\mathrm{CH}_{4}$ is abundant in most of our models where freeze-out occurs - this is because we assume that every neutral and ionized carbon atom that hits the grains hydrogenates to methane which is then released to the gas phase in the second phase of our model. The efficiency of hydrogenation of carbon on grain surfaces is entirely unknown. In the models discussed so far, this efficiency has been taken to be $100 \%$. In order to consider the sensitivity of the results to this assumption we investigate a series of models in which we vary the efficiency with which neutral and ionized carbon, on hitting the grains, hydrogenate to methane.

We also investigate several models where the initial relative elemental abundance of oxygen is halved (still maintaining the same $\mathrm{C} / \mathrm{O}$ ratio). The justification for reducing the initial relative oxygen abundance comes from our rather high choice for the initial relative abundance of cosmic oxygen $\left(4.6 \times 10^{-4}\right)$. This value is close to the upper limit of the interstellar oxygen abundances derived from 14 lines of sight by Meyer et al. (1998, see Table 2).

The new models are B11 (as B2 but with only $10 \%$ of carbon freezing as methane), B12 (as B7 with only $10 \%$ of carbon freezing as methane and half of the original oxygen), B13 (as B9 with only $10 \%$ of carbon freezing as methane and half of the original oxygen), B14 (as B11 with half of the original oxygen), B15 (as B12 but with no carbon freezing as methane).

Figures 4 and 5 show the results from our models, including the new ones, for $\mathrm{H}_{2} \mathrm{CO}$ and $\mathrm{HCO}^{+}$. Again, for all the figures, we also encompass the area of possible observational values for the column densities (lower and upper limit are derived as for $\mathrm{HCO}^{+}$). None of the models succeed in reproducing the low abundance of $\mathrm{H}_{2} \mathrm{CO}$ for long enough: B13 lies within the observational band for $\sim 15$ yrs which may be too short to be significant; B15 on the other hand is still overabundant for the first 100 yrs but only by a factor that varies between $\sim 1.5$ and $\sim 5$. In the context of modelling interstellar chemistry, and the uncertainty in the observations, this is a reasonable agreement. Moreover, if the observed $\mathrm{H}_{2} \mathrm{CO}$ transitions has an opacity of $2-3$, then the fitting with $\mathrm{B} 15$ would be much better. We therefore favour Model B15. $\mathrm{HCO}^{+}$is also well reproduced by Model B15 (Fig. 5) as are SO (Fig. 6) and methanol (Fig. 7). Note that the abundance of SO is slightly underabundant in Model B15. However, the initial relative sulphur abundance is very uncertain and we chose it to be a factor of hundred lower than the solar value - a conventional assumption for dark clouds (Oppenheimer \& Dalgarno 1974; Ruffle et al. 1999). This value may well be in error by a factor of a few, so that we may regard Model B15 as well within the uncertainty for SO.

However, Model B15 does not reproduce the abundances of $\mathrm{HCN}$ and $\mathrm{CN}$. We have examined the chemistry for these two species; we find that at the end of Phase I their abundances are consistent with those observed towards other dense cores (cf. van Dishoeck 1998), although already somewhat too high 

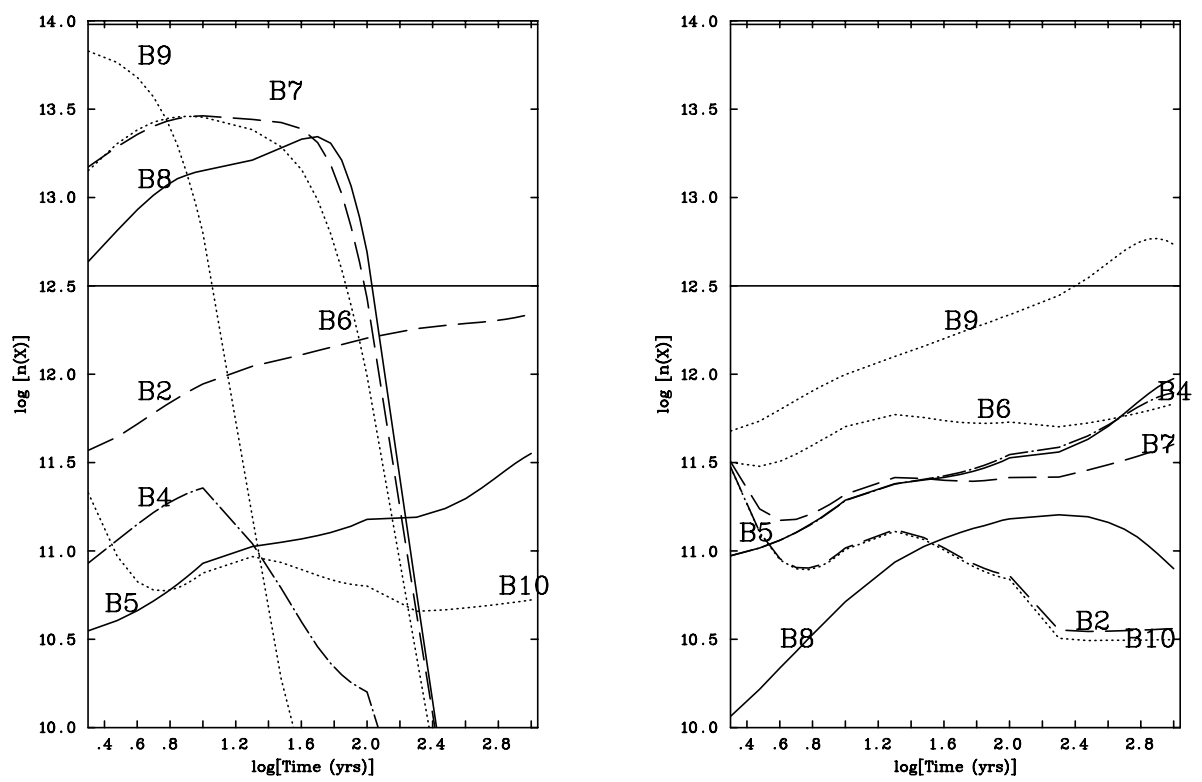

Fig. 3. Column density of $\mathrm{HCO}^{+}$as a function of time at $A_{v}=3.4 \mathrm{mag}$ (left) and $6.2 \mathrm{mag}$ (right) for selected models from Grid B. The two horizontal lines encompass the area of possible observed values for $\mathrm{HCO}^{+}$.

with respect to the observed values for $\mathrm{HH} 2$. This suggests that the (quite conventional) chemical network that produces $\mathrm{CN}$ and $\mathrm{HCN}$ is probably correct. In Phase II, however, $\mathrm{CN}$ quickly increases due to the enhancement of the radiation field, via the reaction:

$\mathrm{NO}+\mathrm{C} \rightarrow \mathrm{CN}+\mathrm{O}$

$\mathrm{CN}$ is then efficiently destroyed by the neutral reactions:

$\mathrm{N}+\mathrm{CN} \rightarrow \mathrm{N}_{2}+\mathrm{C}$

$\mathrm{NH}_{3}+\mathrm{CN} \rightarrow \mathrm{HCN}+\mathrm{NH}_{2}$

therefore enhancing $\mathrm{HCN}$ as well as $\mathrm{CN}$. The first reaction is dominant at early times while the second reaction is dominant after the first 40 years or so. $\mathrm{HCN}$ is therefore a direct consequence of $\mathrm{CN}$ and its destruction (via photodissociation) leads back to CN. Photodissociation of CN does not appear to be very important. This may be due to the rate adopted for this reaction (taken from the UMIST database). We therefore investigate, arbitrarily, the possibility that a faster $\mathrm{CN}$ photodissociation rate would decrease both $\mathrm{CN}$ and $\mathrm{HCN}$, and do this by artificially increasing the rate. We find no substantial difference for the first 100 years. Finally, a possible reason for the discrepancy of predicted abundances of $\mathrm{CN}$ and $\mathrm{HCN}$ versus observed could be that the observed column densities are underabundant because they are optically thick. This will be further investigated by future BIMA observations for $\mathrm{HCN}$.

In conclusion, we can find no reason why the abundances of $\mathrm{HCN}$ and $\mathrm{CN}$ predicted by our models should be much larger than apparently observed. The values computed at the end of Phase I are reasonably consistent with observations of dark clouds, and the introduction of the $\mathrm{HH}$ radiation field will certainly initiate a chemistry that should increase $\mathrm{CN}$ and $\mathrm{HCN}$ production. We conclude that the models suggest the presence of large amounts of $\mathrm{CN}$ and $\mathrm{HCN}$ not currently observed, or

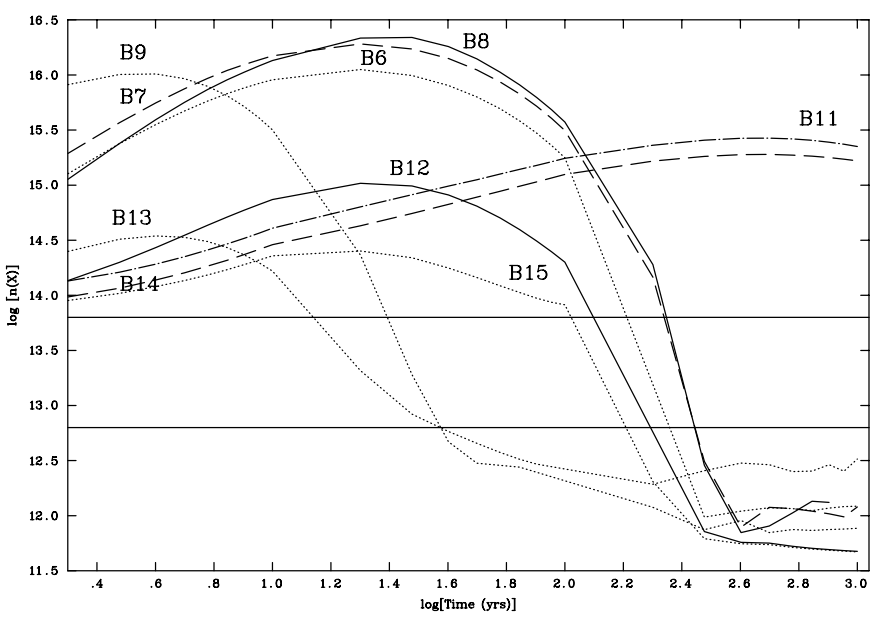

Fig. 4. Fractional abundance of $\mathrm{H}_{2} \mathrm{CO}$ as a function of time for selected models from Grid $\mathrm{B}$ at $A_{V} \sim 3 \mathrm{mag}$.

alternatively that the $\mathrm{HCN}$ and $\mathrm{CN}$ observed column densities are underestimated due to optical depth effects.

\section{Discussion and conclusions}

As a follow up to a molecular line survey toward the quiescent molecular clump ahead of the HH 2 object (Paper I), we have presented here a detailed time-dependent chemical model of the observed clump. There are still considerable uncertainties remaining in the observational results, but we have been able to use these data to restrict the possible chemical models and to identify physical parameters of the clump. Our general conclusions concerning the model are:

- our chemical analysis suggests that the clump ahead of $\mathrm{HH} 2$ was young when it was first irradiated by the $\mathrm{HH}$ object. A long period ( 2 My) at high density is definitely excluded by this study. If future observations of a large 


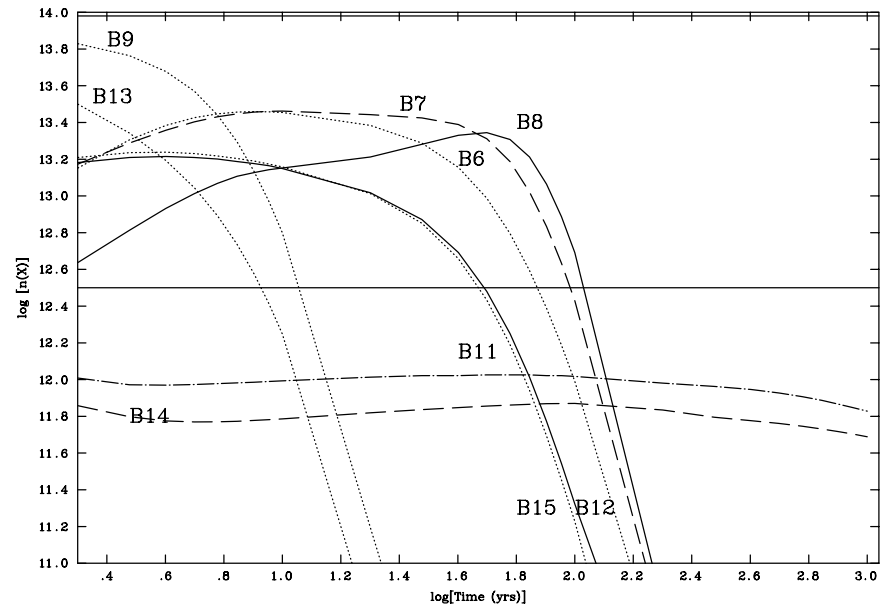

Fig. 5. Fractional abundance of $\mathrm{HCO}^{+}$as a function of time for selected models from Grid B at $A_{V} \sim 3$ mag.

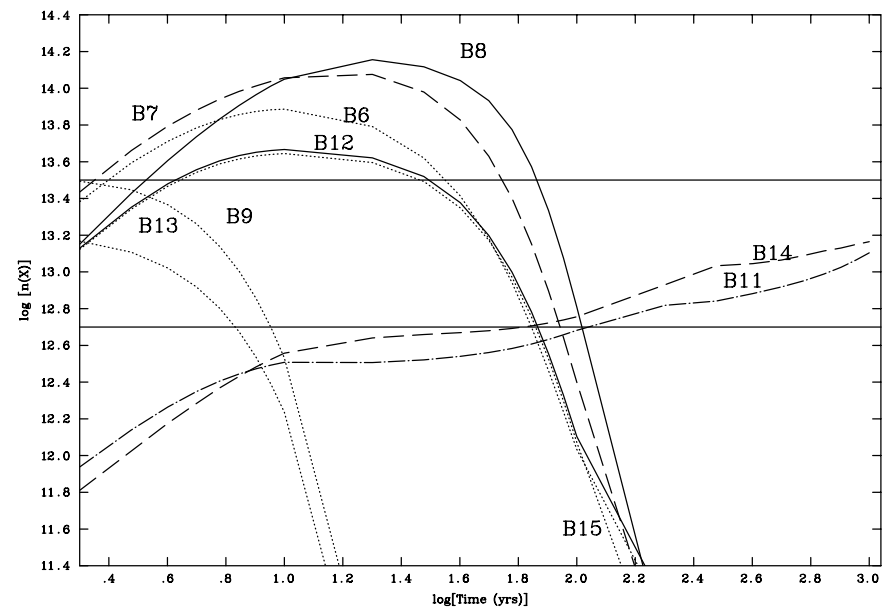

Fig. 6. Fractional abundance of SO as a function of time for selected models from Grid B at $A_{V} \sim 3$ mag.

sample of clumps ahead of HH objects also indicate young material, this may mean that in general clumps in molecular clouds are transient, consistent with current interpretations of molecular clouds by both single dish and array telescopes (see Sect. 1);

- BIMA array observations show a much more complex structure than revealed by single dish CSO observations (see also Hester et al. 1998 where a much more complex structure is suggested). As a consequence, our theoretical clump, modelled on the basis of the SO contour from the $\mathrm{CSO}$, is probably only representative of the small, high density gas just ahead of the $\mathrm{HH}$ object. We find that it is not possible for the clump ahead of $\mathrm{HH} 2$ to have a uniform high density - in fact, a density gradient, where the highest density is $\sim 3 \times 10^{5} \mathrm{~cm}^{-3}$ closest to HH 2 and falling with distance from it, is probably more realistic;

- the characteristic chemical signature seen in HH 2 is of short duration, probably no more than a few hundred years, depending on the density in the clump and the penetration of $\mathrm{HH}$ radiation into it. Thus, secular changes may occur

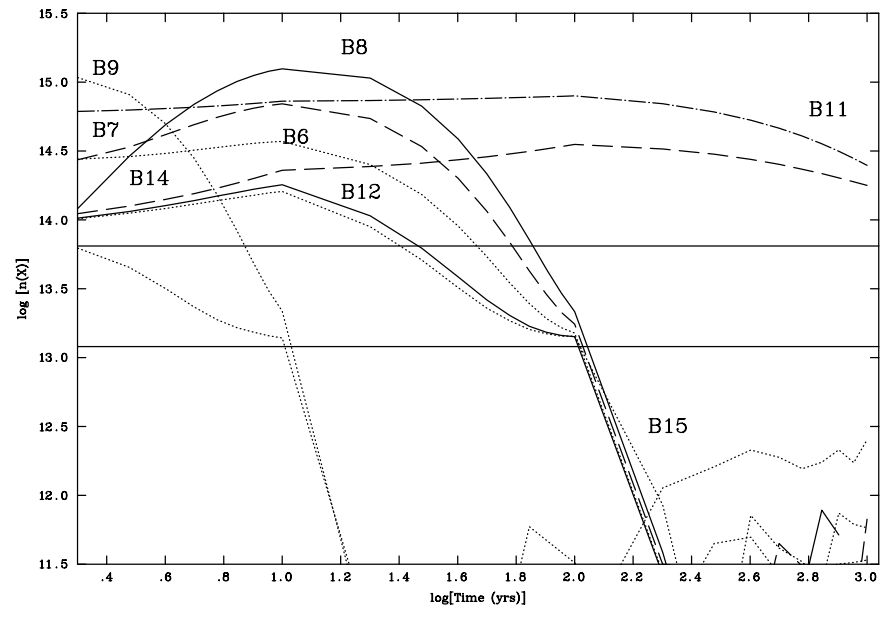

Fig. 7. Fractional abundance of $\mathrm{CH}_{3} \mathrm{OH}$ as a function of time for selected models from Grid B at $A_{V} \sim 3$ mag.

in clumps ahead of $\mathrm{HH}$ objects on astronomically short timescales, possibly short enough to be detectable;

- the peculiar chemistry found ahead of $\mathrm{HH}$ objects is achieved for very particular combinations of ice fraction on grains (at the end of Phase I) and enhanced radiation field caused by the proximity of the $\mathrm{HH}$ object;

- it is difficult to reconcile theoretical predictions with observations if a large quantity of gas phase $\mathrm{CH}_{4}$ is present in Phase II (expected when depletion and hydrogenation occur). We infer that carbon bearing species may not completely hydrogenate as methane when freezing out on grains in Phase I. Note that if this is the case, then Grid A models may be more successful in reproducing the observations; therefore our result on the lifetime of the clump prior to the irradiation of the $\mathrm{HH}$ objects, would be less conclusive.

Despite the difficulties we have found in modelling the chemistry in the clump ahead of $\mathrm{HH} \mathrm{2,} \mathrm{we} \mathrm{believe} \mathrm{that} \mathrm{we} \mathrm{are} \mathrm{able}$ to constrain some of its physical and chemical parameters. In particular:

- the "clump" is probably better described by a two or more density components, with an average density of 1-5 $\times$ $10^{4} \mathrm{~cm}^{-3}$ and a peak density of $\sim 3 \times 10^{5} \mathrm{~cm}^{-3}$;

- the visual extinction along the high density component cannot be higher than $\sim 3.5$ mag;

- the lifetime of the clump after it starts being irradiated cannot be much longer than $\sim 100$ years;

- the radiation field impinging at edge of the high density component of the clump is expected to be in the range of 20 and 1000 Habing, and certainly substantially less than $10^{4}$ Habing;

- the composition of the clump before it is irradiated by the $\mathrm{HH}$ object is probably such that not more than half of the molecular material is depleted on the grains;

- X-ray emission from the HH 2 object does not appear to affect substantially the chemistry of the clump.

Our aim in this work is to reproduce the abundances observed towards the clump ahead of $\mathrm{HH} \mathrm{2}$. Other $\mathrm{HH}$ objects 
may provide a different irradiating field, in both intensity and spectrum, from that of $\mathrm{HH} 2$, and some clumps may have lower/higher density and therefore probably a lower/higher degree of freeze-out than the clump we observe ahead of HH 2. Therefore, the chemistry of clumps ahead of other HH objects may be diverse.

Acknowledgements. RE and JMG acknowledge partial financial support from Spanish MCYT grant AYA2002-00205. RG acknowledges receipt of a PPARC studentship and DAW also wishes to acknowledge receipt of financial support from PPARC.

\section{References}

Choi, M., \& Zhou, S. 1997, ApJ, 477, 754

Davis, C. J., Dent, W. R. F., \& Burnell, S. J. B. 1990, MNRAS, 244, 173

Falle, S. A. E. G., \& Hartquist, T. 2002, MNRAS, 329, 195

Girart, J. M., Rodríguez, L. F., Anglada, G., et al. 1994, ApJ, 435, L145

Girart, J. M., Viti, S., Williams, D. A., Estalella, R., \& Ho, P. T. P. 2002, A\&A, 388, 1004

Haro, G. 1952, ApJ, 115, 572

Herbig, G. H. 1951, ApJ, 113, 697

Hester, J. J., Stapelfeldt, K. R., \& Scowen, P. A. 1998, AJ, 116, 372
Meyer, D. M., Jura, M., \& Cardelli, J. A. 1998, ApJ, 493, 222

Millar, T. J., Farquhar, P. R. A., \& Willacy, K. 1997, A\&AS, 121, 139

Morata, O., Estalella, R., Lopez, R., \& Planesas, P. 1997, MNRAS, 292, 120

Morata, O., Girart, J. M., \& Estalella, R. 2003, A\&A, 397, 181

Myers, P. C., Fuller, G. A., Goodman, A. A., \& Benson, P. J. 1991, ApJ, 376, 561

Nguyen, T. K., Viti, S., \& Williams, D. A. 2002, A\&A, 387, 1083

Oppenheimer, M., \& Dalgarno, A. 1974, ApJ, 187, 231

Pastor, J., Buj, J., Estalella, R., et al. 1991, A\&A, 252, 320

Pravdo, S. H., Fegelson, E. D., Garmire, G., et al. 2001, Science, 413, 708

Raga, A. C., \& Williams, D. A. 2000, A\&A, 358, 701

Rawlings, J. M. C., Hartquist, T. W., Menten, K. M., \& Williams, D. A. 1992, MNRAS, 255, 471

Rudolph, A., \& Welch, W. J. 1988, ApJ, 326, L31

Ruffle, D. P., Hartquist, T. W., Caselli, P., \& Williams, D. A. 1999, MNRAS, 306, 691

Taylor, S. D., Morata, O., \& Williams, D. A. 1996, A\&A, 313, 269

Taylor, S. D., \& Williams, D. A. 1996, MNRAS, 282, 1343

Torrelles, J. M., Rodríguez, L. F., Cantó, J., et al. 1992, ApJ, 396, L95

Torrelles J. M., Gómez, J. F., Ho, P. T. P., et al. 1994, ApJ, 435, 290

van Dishoeck, E. W. 1998, in The Molecular Astrophysics of Stars and Galaxies, ed. T. W. Hartquist, \& D. A. Williams (Clarendon Press, Oxford), 53

Viti, S., \& Williams, D. A. 1999, MNRAS, 310, 517 